\title{
ON MULTIDIMENSIONAL DISCRITE INEQUALITIES AND THEIR APPLICATIONS
}

\author{
B. G. PACHPATTE
}

\begin{abstract}
A.bstract. The aim of the present paper is to establish some new multi-dimensional discrete inequalities which can be used as handy tools in the analysis of a new class of finite difference equations involving functions of several independent variables.
\end{abstract}

\section{Introduction}

In the developments of the theory of finite difference equations and numerical analysis, various types of discrete inequalities are of fundamental importance, since the bounds provided by these inequalities are adequate in many applications. Several papers have recently concerned themselves with a number of new discrete inequalities and their applications to the various types of finite difference equations, see [1-12] and the references given therein. However, in the study of certain new models of multidimensional finite difference equations the bounds provided by the existing results on discrete inequalities are inadequate in applications. It is natural to seek some new discrete inequalities which are readily applicable in order to achieve a diversity of desired goals. Our main objective here is to establish some new multidimensional discrete inequalities which can be used as ready and powerful tools in the qualitative analysis of a new class of finite difference equations involving functions of several independent variables. To illustrate the utility of some of our results we discuss the boundedness, uniqueness and continuous dependence of the solutions of a new class of multidimensional finite difference equations.

\section{Statement of Results}

Before stating the theorems to be proved in this paper, we summarise some basic notations and definitions which will be used throughout our discussion. Let $N_{0}=\{0,1,2, \ldots\}$ and the product $N_{0} \times N_{0} \times \cdots \times N_{0}$ ( $n$ times) be denoted by $N_{0}^{n}$. For all $m>n, m, n \in N_{0}$ and any function $u(s)$ defined on $N_{0}$, we use the usual convention, $\sum_{s=m}^{n} u(s)=0$ and $\prod_{s=m}^{n} u(s)=1$. A point $\left(x_{1}, \ldots, x_{n}\right)$ in $N_{0}^{n}$ is denoted by $x$. For any function $u(x)$ defined on $N_{0}^{n}$, we define the operators

Received. October 3, 1988; revised June 7, 1989. 


$$
\begin{aligned}
& \triangle_{1} u(x)=u\left(x_{1}+1, x_{2}, \ldots, x_{n}\right)-u(x), \\
& \triangle_{2} u(x)=u\left(x_{1}, x_{2}+1, x_{3}, \ldots, x_{n}\right)-u(x), \\
& \vdots \\
& \triangle_{n} u(x)=u\left(x_{1}, \ldots, x_{n-1}, x_{n}+1\right)-u(x) .
\end{aligned}
$$

The operators $L_{j}$ are recursively defined by

$$
L_{0} u(x)=u(x), L_{j} u(x)=p_{j}(x) \triangle_{j} L_{j-1} u(x), j=1, \ldots, n,
$$

$p_{n}(x)=1$, where $u(x)$ and $p_{j}(x)$ are functions defined on $N_{0}^{n}$. For $x, y \in N_{0}^{n}$ and some functions $p_{j}(x)>0, j=1, \ldots, n-1$ and $b(x)$, we set

$$
\begin{aligned}
M\left[x_{1}, \ldots, x_{n}, p_{1}, \ldots, p_{n-1}, b\right]= & \sum_{y_{1}=0}^{x_{1}-1} \frac{1}{p_{1}\left(y_{1}, x_{2}, \ldots, x_{n}\right)} \\
& \ldots \sum_{y_{n-1}=0}^{x_{n-1}-1} \frac{1}{p_{n-1}\left(y_{1}, \ldots, y_{n-1}, x_{n}\right)} \sum_{y_{n}=0}^{x_{n}-1} b(y),
\end{aligned}
$$

and

$$
\begin{aligned}
H\left[y_{1}, x_{2}, \ldots, x_{n}, p_{1}, \ldots, p_{n-1}, b\right]= & \frac{1}{p_{1}\left(y_{1}, x_{2}, \ldots, x_{n}\right)} \sum_{y_{2}=0}^{x_{2}-1} \frac{1}{p_{2}\left(y_{1}, y_{2}, x_{3}, \ldots, x_{n}\right)} \\
& \ldots \sum_{y_{n-1}=0}^{x_{n-1}-1} \frac{1}{p_{n-1}\left(y_{1}, \ldots, y_{n-1}, x_{n}\right)} \sum_{y_{n}=0}^{x_{n}-1} b(y) .
\end{aligned}
$$

For convenience we list the following hypotheses.

(A1) $u(x)$ and $h(x)$ are real-valued nonnegative functions defined for $x \in N_{0}^{n}$.

(A2) $p_{j}(x), j=1, \ldots, n-1$, are real-valued positive functions defined for $x \in N_{0}^{n}$.

(A3) $a(x)$ is real-valued, positive and nondecreasing function in all the components $x_{1}, \ldots, x_{n}$ of $x \in N_{0}^{n}$.

(A4) $u(x) \geq u_{0} \geq 0, u_{0}$ is a constant, $h(x) \geq 0$ are real-valued functions defined for $x \in N_{0}^{n}$.

(A5) $g(u)$ is continuous, nondecreasing real-valued function defined on an interval $I=$ $\left[u_{0}, \infty\right), u_{0} \geq 0$ is a constant and $g(u)>0$ on $\left(u_{0}, \infty\right), g\left(u_{0}\right)=0$.

(A6) $q_{j}(x), j=1, \ldots, n-1$, are real-valued positive functions defined for $x \in N_{0}^{n}$.

(A7) $W(u)$ is continuous, nondecreasing and submultiplicative real-valued function defined on an interval $I$, and $W(u)>0$ on $\left(u_{0}, \infty\right), W\left(u_{0}\right)=0$.

Our main result is established in the following 
Theorem 1. Suppose (A1) and (A2) are true. If

$$
u(x) \leq c+M\left[x_{1}, \ldots, x_{n}, p_{1}, \ldots, p_{n-1}, h u\right]
$$

for $x \in N_{0}^{n}$, where $c$ is a nonnegative constant, then

$$
u(x) \leq c \prod_{y_{1}=0}^{x_{1}-1}\left[1+H\left[y_{1}, x_{2}, \ldots, x_{n}, p_{1}, \ldots, p_{n-1}, b\right]\right],
$$

for $x \in N_{0}^{n}$.

A useful and slightly different version of Theorem 1 is embodied in the following theorem.

Theorem 2. Suppose (A1), (A2) and (A3) are true. If

$$
u(x) \leq a(x)+M\left[x_{1}, \ldots, x_{n}, p_{1}, \ldots, p_{n-1}, h u\right],
$$

for $x \in N_{0}^{n}$, then

$$
u(x) \leq a(x) \prod_{y_{1}=0}^{x_{1}-1}\left[1+H\left[y_{1}, x_{2}, \ldots, x_{n}, p_{1}, \ldots, p_{n-1}, h\right]\right]
$$

for $x \in N_{0}^{n}$.

Another interesting and useful discrete inequality is established in the following theorem.

Theorem 3. Suppose (A2), (A4) and (A5) are true. If

$$
u(x) \leq c+M\left[x_{1}, \ldots, x_{n}, p_{1}, \ldots, p_{n-1}, h g(u)\right]
$$

for $x \in N_{0}^{n}$, where $c$ is a nonnegative constant, then for $x_{j}, x_{j}^{*}$ and $0 \leq x_{j} \leq x_{j}^{*}, j=$ $1, \ldots, n$,

$$
u(x) \leq G^{-1}\left[G(c)+M\left[x_{1}, \ldots, x_{n}, p_{1}, \ldots, p_{n-1}, h\right]\right]
$$

where

$$
G(r)=\int_{r_{0}}^{r} \frac{d s}{g(s)}, r \geq u_{0} \text { with } r_{0}>u_{0}
$$

$G^{-1}$ is the inverse of $G$ and $x_{j}^{*} \in N_{0}, j=1, \ldots, n$, are chosen so that

$$
G(c)+M\left[x_{1}, \ldots, x_{n}, p_{1}, \ldots, p_{n-1}, h\right] \in \operatorname{Dom}\left(G^{-1}\right)
$$

for all $x_{j}$ with $0 \leq x_{j} \leq x_{j}^{*}, j=1, \ldots, n$ for $x_{j}, x_{j}^{*} \in N_{0}$. 
We next establish the following more general inequality which may be convenient in some applications.

Theorem 4. Suppose (A1),(A2), (A6) and (A7) are true. If

$$
\begin{aligned}
u(x) \leq c & +M\left[x_{1}, \ldots, x_{n}, p_{1}, \ldots, p_{n-1}, h u\right] \\
& +M\left[x_{1}, \ldots, x_{n}, q_{1}, \ldots, q_{n-1}, k W(u)\right]
\end{aligned}
$$

for $x \in N_{0}^{n}$, where $c$ is a nonnegative constant and $k(x)$ is a realvalued nonnegative function defined for $x \in N_{0}^{n}$, then for $x_{j}, x_{j}^{* *} \in N_{0}$ and $0 \leq x_{j} \leq x_{j}^{* *}, j=1, \ldots, n$,

$$
u(x) \leq Q(x) \Omega^{-1}\left[\Omega(c)+M\left[x_{1}, \ldots, x_{n}, q_{1}, \ldots, q_{n-1}, k W(Q)\right]\right]
$$

where

$$
Q(x)=\prod_{y_{1}=0}^{x_{1}-1}\left[1+H\left[y_{1}, x_{2}, \ldots, x_{n}, p_{1}, \ldots, p_{n-1}, h\right]\right]
$$

and

$$
\Omega(r)=\int_{r_{0}}^{r} \frac{d s}{W(s)}, r \geq u_{0} \text { with } r_{0}>u_{0}
$$

$\Omega^{-1}$ is the inverse of $\Omega$ and $x_{j}^{* * *} \in N_{0}, j=1, \ldots, n$ are chosen so that

$$
\Omega(c)+M\left[x_{1}, \ldots, x_{n}, q_{1}, \ldots, q_{n-1}, k W(Q)\right] \in \operatorname{Dom}\left(\Omega^{-1}\right),
$$

for all $x_{j}$ with $0 \leq x_{j} \leq x_{j}^{* *}, j=1, \ldots, n$ for $x_{j}, x_{j}^{* *} \in N_{0}$.

3. Proofs of Theorems $1-4$

In order to establish the inequality (1) in Theorem 1 , we first assume that $c>0$ and define a function $z(x)$ by

$$
z(x)=c+M\left[x_{1}, \ldots, x_{n}, p_{1}, \ldots, p_{n-1}, h u\right]
$$

From (12) it is easy to observe that

$$
\begin{aligned}
L_{1} z(x)= & \sum_{y_{2}=0}^{x_{2}-1} \frac{1}{p_{2}\left(x_{1}, y_{2}, x_{3}, \ldots, x_{n}\right)} \\
& \ldots \sum_{y_{n-1}=0}^{x_{n-1}-1} \frac{1}{p_{n-1}\left(x_{1}, y_{2}, \ldots, y_{n-1}, x_{n}\right)} \\
& \cdot \sum_{y_{n}=0}^{x_{n}-1} h\left(x_{1}, y_{2}, \ldots, y_{n}\right) u\left(x_{1}, y_{2}, \ldots, y_{n}\right),
\end{aligned}
$$




$$
\begin{aligned}
L_{2} z(x)= & \sum_{y_{3}=0}^{x_{3}-1} \frac{1}{p_{3}\left(x_{1}, x_{2}, y_{3}, x_{4}, \ldots, x_{n}\right)} \\
& \ldots \sum_{y_{n-1}=0}^{x_{n-1}-1} \frac{1}{p_{n-1}\left(x_{1}, x_{2}, y_{3}, \ldots, y_{n-1}, x_{n}\right)} \\
& \cdot \sum_{y_{n}=0}^{x_{n}-1} h\left(x_{1}, x_{2}, y_{3}, \ldots, y_{n}\right) u\left(x_{1}, x_{2}, y_{3}, \ldots, y_{n}\right),
\end{aligned}
$$

and continuing in this way we obtain

$$
\begin{aligned}
L_{n-2} z(x)= & \sum_{y_{n-1}=0}^{x_{n-1}-1} \frac{1}{p_{n-1}\left(x_{1}, \ldots, x_{n-2}, y_{n-1}, x_{n}\right)} \\
& \cdot \sum_{y_{n}=0}^{x_{n}-1} h\left(x_{1}, \ldots, x_{n-2}, y_{n-1}, y_{n}\right) u\left(x_{1}, \ldots, x_{n-2}, y_{n-1}, y_{n}\right),
\end{aligned}
$$

$$
\begin{aligned}
L_{n-1} z(x) & =\sum_{y_{n}=0}^{x_{n}-1} h\left(x_{1}, \ldots, x_{n-1}, y_{n}\right) u\left(x_{1}, \ldots, x_{n-1}, y_{n}\right) \\
L_{n} z(x) & =h(x) u(x) .
\end{aligned}
$$

Using the fact that $u(x) \leq z(x)$ in (17) we have

$$
L_{n} z(x) \leq h(x) z(x) \text {. }
$$

From the definition of $z(x)$ we observe that $z(x) \leq z\left(x_{1}, \ldots, x_{n-1}, x_{n}+1\right)$ for all $x_{j} \in$ $N_{0}, 1 \leq j \leq n$. Using this fact in (18) we see that

$$
\frac{L_{n-1} z\left(x_{1}, \ldots, x_{n-1}, x_{n}+1\right)}{z\left(x_{1}, \ldots, x_{n-1}, x_{n}+1\right)}-\frac{L_{n-1} z(x)}{z\left(x_{1}, \ldots, x_{n-1}, x_{n}+1\right)} \leq h(x) .
$$

From (19) and the fact that $L_{n-1} z(x) \geq 0$ from (16), we observe that

$$
\frac{L_{n-1} z\left(x_{1}, \ldots, x_{n-1}, x_{n}+1\right)}{z\left(x_{1}, \ldots, x_{n-1}, x_{n}+1\right)}-\frac{L_{n-1} z(x)}{z(x)} \leq h(x) .
$$

Now keeping the components $x_{1}, \ldots, x_{n-1}$ of $x$ fixed in (20), set $x_{n}=y_{n}$ and sum over $y_{n}=0,1,2, \ldots, x_{n}-1$, and use the fact that $L_{n-1} z\left(x_{1}, \ldots, x_{n-1}, 0\right)=0$ from (16), to obtain the estimate

$$
\frac{L_{n-1} z(x)}{z(x)} \leq \sum_{y_{n}=0}^{x_{n}-1} h\left(x_{1}, \ldots, x_{n-1}, y_{n}\right)
$$

From (21) and in view of the facts that $z(x) \leq z\left(x_{1}, \ldots, x_{n-2}, x_{n-1}+1, x_{n}\right)$ for all $x_{j} \in N_{0}, 1 \leq j \leq n$ and $L_{n-2} z(x) \geq 0$, we observe that

$$
\begin{aligned}
& \frac{L_{n-2} z\left(x_{1}, \ldots, x_{n-2}, x_{n-1}+1, x_{n}\right)}{z\left(x_{1}, \ldots, x_{n-2}, x_{n-1}+1, x_{n}\right)}-\frac{L_{n-2} z(x)}{z(x)} \\
& \leq \frac{1}{p_{n-1}(x)} \sum_{y_{n}=0}^{x_{n}-1} h\left(x_{1}, \ldots, x_{n-1}, y_{n}\right) .
\end{aligned}
$$


Keeping the components $x_{1}, \ldots, x_{n-2}, x_{n}$ of $x$ fixed in (22), set $x_{n-1}=y_{n-1}$ and sum over $y_{n-1}=0,1,2, \ldots, x_{n-1}-1$, and use the fact that $L_{n-2} z\left(x_{1}, \ldots, x_{n-2}, 0, x_{n}\right)=0$ from (15), to obtain the estimate

$$
\begin{aligned}
\frac{L_{n-2} z(x)}{z(x)} \leq & \sum_{y_{n-1}=0}^{x_{n-1}-1} \frac{1}{p_{n-1}\left(x_{1}, \ldots, x_{n-2}, y_{n-1}, x_{n}\right)} \\
& \cdot \sum_{y_{n}=0}^{x_{n}-1} h\left(x_{1}, \ldots, x_{n-2}, y_{n-1}, y_{n}\right) .
\end{aligned}
$$

Proceeding in this way we obtain the estimate

$$
\begin{aligned}
\frac{L_{1} z(x)}{z(x)} \leq & \sum_{y_{2}=0}^{x_{2}-1} \frac{1}{p_{2}\left(x_{1}, y_{2}, x_{3}, \ldots, x_{n}\right)} \\
& \ldots \sum_{y_{n-1}=0}^{x_{n-1}-1} \frac{1}{p_{n-1}\left(x_{1}, y_{2}, \ldots, y_{n-1}, x_{n}\right)} \\
& \cdot \sum_{y_{n}=0}^{x_{n}-1} h\left(x_{1}, y_{2}, \ldots, y_{n}\right) .
\end{aligned}
$$

From (24) we see that

$$
\begin{aligned}
z\left(x_{1}+1, x_{2}, \ldots, x_{n}\right) \leq z(x)[1+ & \frac{1}{p_{1}(x)} \sum_{y_{2}=0}^{x_{2}-1} \frac{1}{p_{2}\left(x_{1}, y_{2}, x_{3}, \ldots, x_{n}\right)} \\
& \cdots \sum_{y_{n-1}=0}^{x_{n-1}-1} \frac{1}{p_{n-1}\left(x_{1}, y_{2}, \ldots, y_{n-1}, x_{n}\right)} \\
& \left.\cdot \sum_{y_{n}=0}^{x_{n}-1} h\left(x_{1}, y_{2}, \ldots, y_{n}\right)\right]
\end{aligned}
$$

Now keeping the components $x_{2}, \ldots, x_{n}$ of $x$ fixed in (25), set $x_{1}=y_{1}$ and substitute $y_{1}=0,1,2, \ldots, x_{1}-1$ successively and use the fact that $z\left(0, x_{2}, \ldots, x_{n}\right)=0$ from (12), to obtain the estimate

$$
z(x) \leq c \prod_{y_{1}=0}^{x_{1}-1}\left[1+H\left[y_{1}, x_{2}, \ldots, x_{n}, p_{1}, \ldots, p_{n-1}, h\right]\right] .
$$

Substituting this bound on $z(x)$ on the right side of (1) we obtain the inequality (2).

Now suppose $c=0$. Then from (1) we see that the inequality

$$
u(x) \leq \epsilon+M\left[x_{1}, \ldots, x_{n}, p_{1}, \ldots, p_{n-1}, h u\right],
$$


holds for every arbitrary positive number $\epsilon$ and $x \in N_{0}^{n}$, which by the above argument yields the estimate

$$
u(x) \leq \epsilon \prod_{y_{1}=0}^{x_{1}-1}\left[1+H\left[y_{1}, x_{2}, \ldots, x_{n}, p_{1}, \ldots, p_{n-1}, h\right]\right] .
$$

Since $u(x) \geq 0$ and $\varepsilon>0$ is arbitrary number independent of $x$, then from (27) it follows that $u(x)=0$. This completes the proof of Theorem 1 .

Since $a(x)$ is positive and nondecreasing in each component $x_{1}, \ldots, x_{n}$ of $x$, we observe from (3) that

$$
\frac{u(x)}{a(x)} \leq 1+M\left[x_{1}, \ldots, x_{n}, p_{1}, \ldots, p_{n-1}, h \frac{u}{a}\right] .
$$

Now an application of Theorem 1 yields the required bound in (4) and the proof of Theorem 2 is complete.

In order to establish the inequality (6) in Theorem 3, let $\epsilon>0$ and $u_{\varepsilon}(x)=u(x)+\epsilon \geq$ $u_{0}$ for all $x \in N_{0}^{n}$. Then from (5) we see that

$$
\begin{aligned}
u_{\epsilon}(x) & \leq c+\epsilon+M\left[x_{1}, \ldots, x_{n}, p_{1}, \ldots, p_{n-1}, h g\left(u_{\epsilon}-\epsilon\right)\right] \\
& \leq c+\epsilon+M\left[x_{1}, \ldots, x_{n}, p_{1}, \ldots, p_{n-1}, h g\left(u_{\epsilon}\right)\right] .
\end{aligned}
$$

Define a function $z(x)$ by

$$
z(x)=c+\epsilon+M\left[x_{1}, \ldots, x_{n}, p_{1}, \ldots, p_{n-1}, h g\left(u_{\epsilon}\right)\right] .
$$

From (29) it is easy to observe that

$$
\begin{aligned}
L_{1} z(x)= & \sum_{y_{2}=0}^{x_{2}-1} \frac{1}{p_{2}\left(x_{1}, y_{2}, x_{3}, \ldots, x_{n}\right)} \\
& \cdots \sum_{y_{n-1}=0}^{x_{n-1}-1} \frac{1}{p_{n-1}\left(x_{1}, y_{2}, \ldots, y_{n-1}, x_{n}\right)} \\
& \cdot \sum_{y_{n}=0}^{x_{n}-1} h\left(x_{1}, y_{2}, \ldots, y_{n}\right) g\left(u_{\epsilon}\left(x_{1}, y_{2}, \ldots, y_{n}\right)\right), \\
L_{2} z(x)= & \sum_{y_{3}=0}^{x_{3}-1} \frac{1}{p_{3}\left(x_{1}, x_{2}, y_{3}, x_{4}, \ldots, x_{n}\right)} \\
& \quad \cdot \sum_{y_{n}=0}^{x_{n-1}-1} \frac{1}{x_{n-1}=0} h\left(x_{1}, x_{2}, y_{3}, \ldots, y_{n}\right) g\left(u_{\epsilon}\left(x_{1}, x_{2}, y_{3}, \ldots, y_{n}\right)\right),
\end{aligned}
$$


and continuing in this way we obtain

$$
\begin{aligned}
L_{n-2} z(x)= & \sum_{y_{n-1}=0}^{x_{n-1}-1} \frac{1}{p_{n-1}\left(x_{1}, \ldots, x_{n-2}, y_{n-1}, x_{n}\right)} \\
& \cdot \sum_{y_{n}=0}^{x_{n}-1} h\left(x_{1}, \ldots, x_{n-2}, y_{n-1}, y_{n}\right) g\left(u_{\epsilon}\left(x_{1}, \ldots, x_{n-2}, y_{n-1}, y_{n}\right)\right),
\end{aligned}
$$

$$
\begin{aligned}
L_{n-1} z(x) & =\sum_{y_{n}=0}^{x_{n}-1} h\left(x_{1}, \ldots, x_{n-1}, y_{n}\right) g\left(u_{\epsilon}\left(x_{1}, \ldots, x_{n-1}, y_{n}\right)\right), \\
L_{n} z(x) & =h(x) g\left(u_{\epsilon}(x)\right) .
\end{aligned}
$$

Using the fact that $u_{\epsilon}(x) \leq z(x)$ in $(34)$ we have

$$
L_{n} z(x) \leq h(x) g(z(x)) .
$$

From the definition of $z(x)$ in (29), we observe that $z(x) \leq z\left(x_{1}, \ldots, x_{n-1}, x_{n}+1\right)$ for $x \in N_{0}^{n}$. Using this and the fact $L_{n-1} z(x) \geq 0$ from (33), we observe from (35) that

$$
\frac{L_{n-1} z\left(x_{1}, \ldots, x_{n-1}, x_{n}+1\right)}{g\left(z\left(x_{1}, \ldots, x_{n-1}, x_{n}+1\right)\right)}-\frac{L_{n-1} z(x)}{g(z(x))} \leq h(x) .
$$

Now by following exactly the same steps as in the proof of Theorem 1 below the inequality (20) upto the inequality (24) with suitable changes, we obtain

$$
\begin{aligned}
& \frac{L_{1} z(x)}{g(z(x)) \leq} \sum_{y_{2}=0}^{x_{2}-1} \frac{1}{p_{2}\left(x_{1}, y_{2}, x_{3}, \ldots, x_{n}\right)} \\
& \\
& \quad \ldots \sum_{y_{n-1}=0}^{x_{n-1}-1} \frac{1}{p_{n-1}\left(x_{1}, y_{2}, \ldots, y_{n-1}, x_{n}\right)} \\
& \cdot \sum_{y_{n}=0}^{x_{n}-1} h\left(x_{1}, y_{2}, \ldots, y_{n}\right) .
\end{aligned}
$$

From (7) and (37) we have

$$
\begin{aligned}
& G\left(z\left(x_{1}+1, x_{2}, \ldots, x_{n}\right)\right)-G(z(x)) \\
& =\int_{z(x)}^{z\left(x_{1}+1, x_{2}, \ldots, x_{n}\right)} \frac{d s}{g(s)} \\
& \leq \frac{\triangle_{1} z(x)}{g(z(x))} \\
& \leq \frac{1}{p_{1}(x)} \sum_{y_{2}=0}^{x_{2}-1} \frac{1}{p_{2}\left(x_{1}, y_{2}, x_{3}, \ldots, x_{n}\right)}
\end{aligned}
$$




$$
\begin{aligned}
& \cdots \sum_{y_{n-1}=0}^{x_{n-1}-1} \frac{1}{p_{n-1}\left(x_{1}, y_{2}, \ldots, y_{n-1}, x_{n}\right)} \\
& \cdot \sum_{y_{n}=0}^{x_{n}-1} h\left(x_{1}, y_{2}, \ldots, y_{n}\right) .
\end{aligned}
$$

Now keeping the components $x_{2}, \ldots, x_{n}$ of $x$ fixed in (38), set $x_{1}=y_{1}$ and sum over $y_{1}=0,1,2, \ldots, x_{1}-1$ and use the fact that $z\left(0, x_{2}, \ldots, x_{n}\right)=c+\epsilon$, to obtain the estimate

$$
G(z(x)) \leq G(c+\epsilon)+M\left[x_{1}, \ldots, x_{n}, p_{1}, \ldots, p_{n-1}, h\right] .
$$

The bound in (6) now follows by substituting the bound for $z(x)$ from (39) in (28) and letting $\epsilon \rightarrow 0$ and the proof of Theorem 3 is complete.

In order to prove the inequality (9) in Theorem 4, let $\epsilon>0$ and $u_{\varepsilon}(x)=u(x)+\epsilon \geq u_{0}$ for $x \in N_{0}^{n}$. Then from (8) we see that

$$
\begin{gathered}
u_{\varepsilon}(x) \leq c+\epsilon+M\left[x_{1}, \ldots, x_{n}, p_{1}, \ldots, p_{n-1}, h\left(u_{\epsilon}-\epsilon\right)\right] \\
+M\left[x_{1}, \ldots, x_{n}, q_{1}, \ldots, q_{n-1}, k W\left(u_{\epsilon}-\epsilon\right)\right] \\
\leq c+\epsilon+M\left[x_{1}, \ldots, x_{n}, p_{1}, \ldots, p_{n-1}, h u_{\epsilon}\right] \\
+M\left[x_{1}, \ldots, x_{n}, q_{1}, \ldots, q_{n-1}, k W\left(u_{\epsilon}\right)\right] .
\end{gathered}
$$

Define

$$
a(x)=c+\epsilon+M\left[x_{1}, \ldots, x_{n}, q_{1}, \ldots, q_{n-1}, k W\left(u_{\epsilon}\right)\right],
$$

then $(40)$ can be stated as

$$
u_{\epsilon}(x) \leq a(x)+M\left[x_{1}, \ldots, x_{n}, p_{1}, \ldots, p_{n-1}, h u_{\epsilon}\right] .
$$

Since $a(x)$ is positive and nondecreasing function in all the components $x_{1}, \ldots, x_{n}$ of $x$, we have from Theorem 2

$$
u_{\epsilon}(x) \leq Q(x) a(x),
$$

where $Q(x)$ is as defined in (10). Since $W$ is submultiplicative, we have

$$
W\left(u_{\epsilon}(x)\right) \leq W(Q(x)) W(a(x)) .
$$

From (41) and (43) we have

$$
a(x) \leq c+\epsilon+M\left[x_{1}, \ldots, x_{n}, q_{1}, \ldots, q_{n-1}, k W(Q) W(a)\right] .
$$

Now by following the proof of Theorem 3 with suitable modifications we obtain

$$
a(x) \leq \Omega^{-1}\left[\Omega(c+\epsilon)+M\left[x_{1}, \ldots, x_{n}, q_{1}, \ldots, q_{n-1}, k W(Q)\right]\right] .
$$

The desired bound in (9) now follows by substituting (44) in (42) and letting $\epsilon \rightarrow 0$. This completes the proof of Theorem 4 . 


\section{Some applications}

In this section, we present some applications to a new class of finite difference equations involving several independent variables, to convey the importance of our results to the literature. These applications are not stated as theorems so as to obscure the main ideas with tehnical details.

Example 1. As a first application, we obtain a bound on the solution of a nonlinear finite difference equation of the form

$$
L_{n} u(x)=f(x, u(x)),
$$

with the given boundary conditions at $x_{1}=0, \ldots, x_{n}=0$, where $L_{n} u(x)$ is the operator as defined earlier, $p_{j}(x), j=1, \ldots, n$ are real-valued positive functions defined on $N_{0}^{n}$ with $p_{n}(x)=1, f: N_{0}^{n} \times R \rightarrow R$, where $R$ denotes the set of real numbers. We assume that

$$
|f(x, u)| \leq h(x)|u|,
$$

where $h(x)$ is a real-valued nonnegative function defined for $x \in N_{0}^{n}$. By using the given boundary conditions, equation (45) can be represented by the equivalent equation

$$
u(x)=b(x)+M\left[x_{1}, \ldots, x_{n}, p_{1}, \ldots, p_{n-1}, f(u)\right],
$$

where $b(x)$ depends on the functions $p_{j}(x), j=1, \ldots, n-1$ and on the given boundary conditions, and in (47) we have set $f(x, u(x))=f(u)$. Suppose that

$$
|b(x)| \leq k,
$$

where $k$ is a nonnegative constant. Using (46) and (48) in (47) we have

$$
|u(x)| \leq k+M\left[x_{1}, \ldots, x_{n}, p_{1}, \ldots, p_{n-1}, h|u|\right] .
$$

Now an application of Theorem 1 yields the bound on the solution $u(x)$ of (45) in terms of the known functions.

Example 2. As a second application, we shall discuss the uniqueness of the solutions of (45) with the given boundary conditions. We assume that the function $f$ in (45) satisfies

$$
|f(x, u)-f(x, \bar{u})| \leq h(x)|u-\bar{u}|,
$$

where $h(x)$ is as in Example 1. The equation (45) with the given boundary conditions is equivalent to the equation (47). Then for any two solutions $u$ and $\bar{u}$ of (47) we have

$$
|u(x)-\bar{u}(x)| \leq \epsilon+M\left[x_{1}, \ldots, x_{n}, p_{1}, \ldots, p_{n-1}, h|u-\bar{u}|\right],
$$

where $\epsilon>0$ is arbitrary constant. The assumption (49) is used to get the inequality in (50). Now an application of Theorem 1 yields

$$
|u(x)-\bar{u}(x)| \leq \epsilon \prod_{y_{1}=0}^{x_{1}-1}\left[1+H\left[y_{1}, x_{2}, \ldots, x_{n}, p_{1}, \ldots, p_{n-1}, h\right]\right] .
$$

Since $\epsilon>0$ is arbitrary we have $u=\bar{u}$ i.e. there is at most one solution of the equation (45) with the given boundary conditions. 
Example 3. Our third application is an example of continuous dependence of the solution on the equation and boundary data. Consider the equation (45) with the given boundary conditions in Example 1 and the equation

$$
L_{n} z(x)=F(x, z(x)) \text {, }
$$

with the given boundary conditions at $x_{1}=0, \ldots, x_{n}=0$, where $L_{n} z(x)$ is as defined earlier in Example 1, $F: N_{0}^{n} \times R \rightarrow R$. The equation (45) with the given boundary conditions and the equation (51) with the given boundary conditions are equivalent to equations (47) and

$$
z(x)=\bar{b}(x)+M\left[x_{1}, \ldots, x_{n}, p_{1}, \ldots, p_{n-1}, F(z)\right]
$$

where $\bar{b}(x)$ depends on the functions $p_{j}(x), j=1, \ldots, n-1$ and the given boundary data and in (52) we have set $F(x, z(x))=F(z)$. From (47) and (52) we have

$$
u(x)-z(x)=b(x)-\bar{b}(x)+M\left[x_{1}, \ldots, x_{n}, p_{1}, \ldots, p_{n-1},\{f(u)-F(u)\}\right] .
$$

Suppose that the function $f$ in (45) satisfies the condition (49) and further we assume that

$$
\begin{gathered}
|b(x)-\bar{b}(x)| \leq \epsilon \\
M\left[x_{1}, \ldots, x_{n}, p_{1}, \ldots, p_{n-1},|f(z)-F(z)|\right] \leq \epsilon,
\end{gathered}
$$

where $\epsilon>0$ is arbitrary constant. By subtracting and adding $f(z)$ in the braces on the right side of equation (53) and using (49), (54), (55) we obtain

$$
\begin{aligned}
|u(x)-z(x)| \leq|b(x)-\bar{b}(x)|+M\left[x_{1}, \ldots, x_{n}, p_{1}, \ldots, p_{n-1},|f(u)-f(z)|\right] & \\
& \quad+M\left[x_{1}, \ldots, x_{n}, p_{1}, \ldots, p_{n-1},|f(z)-F(z)|\right] \\
\leq & 2 \epsilon+M\left[x_{1}, \ldots, x_{n}, p_{1}, \ldots, p_{n-1}, h|u-z|\right] .
\end{aligned}
$$

Now an application of Theorem 1 yields

$$
|u(x)-z(x)| \leq 2 \epsilon\left\{\prod_{y_{1}=0}^{x_{1}-1}\left[1+H\left[y_{1}, x_{2}, \ldots, x_{n}, p_{1}, \ldots, p_{n-1}, h\right]\right]\right\} .
$$

If $h(x)$ is bounded on some compact set $0 \leq x_{j} \leq \bar{x}_{j}, j=1, \ldots, n-1$ and $x_{j}, \bar{x}_{j} \in N_{0}$, then the quantity in braces on the right in (56) is bounded by some constant $B$ on the set $0 \leq x_{j} \leq \bar{x}_{j}$. Therefore $|u(x)-z(x)| \leq 2 B \epsilon$ on the set $0 \leq x_{j} \leq \bar{x}_{j}$; so that the solution $u(x)$ of (45) with the given boundary conditions depends continuously on $f$ and the boundary data. If $\epsilon \rightarrow 0$, then $|u(x)-z(x)| \rightarrow 0$ on this set. 


\section{References}

[1] Y. V. Bykov and V. G. Linenko, "The stability of solutions of total difference equations (Russian)," Differentsial'nye Uravnenjya 9 (1973), 349-354.

[2] V. B. Demidovich, "A stability test for difference equations (Russian)," Differentsial'nye Uravenjya 5 (1969), 1247-1255.

[3] P. Henrici, Discrete variable methods in ordinary differential equation, Wiley, New York, 1962.

[4] T. E. Hull and W. A. J. Luxemburg, "Numerical methods and existence theorems for ordinary differential equations," Numer. Math. 2 (1960), 30-41.

[5] G. S. Jones, "Fundamental inequalities for discrete and discontinuous functional equations," J. Soc. Indust. Appl. Math. 12 (1964), 43-57.

[6] L. V. Masolockaja, "Stability of difference equations (Russian)," Differentsial'nye Uravenjya 34 (1967), 1976-1983.

[7] B. G. Pachpatte, "Finite difference inequalities and an extension of Lyapunov's method," Michigan Math. J. 18 (1971), 385-391.

[8] B. G. Pachpatte, "On some fundamental finite difference inequalities," Univ. Beograd Publ. Elek. Fak. Ser. Mat. Fiz. No.577 No.598 (1977),65-73.

[9] B. G. Pachpatte, "A note on some fundamental discrete inequalities of the Gronwall-Bellman type," Bull. Inst. Math. Acad. Sinica 5 (1977), 121-128.

[10] B. G. Pachpatte, "On some fundamental discrete inequalities in two independent variables," Tamkang J. Math. 12 (1982), 21-33.

[11] S. Sugiyama, "On the stability problems of difference equations," Bull. Sci. Enger. Research Lab. Waseda Univ. 45 (1969), 140-144.

[12] P. Talpalaru, "On finite time stability of difference systems," An. Sti. Univ., Al. I. Cuz" Iasi 20 (1974), 389-394.

Department of Mathematics and statistics, Marathwada University, Aurangabad 431004 (Maharashtra), India. 